\title{
Do Benzodiazepines Still Need to be Used?
}

\author{
Michel Bourin* \\ Neurobiology of anxiety and depression, University of Nantes, France
}

Submission: August 10, 2017; Published: August 30, 2017

*Corresponding author: Michel Bourin, Neurobiology of anxiety and depression, University of Nantes, 98, rue Joseph Blanchart 44100 Nantes, France, Email: michel.bourin@univ-nantes.fr

\begin{abstract}
Summary
In recent years, the use of benzodiazepines has decreased significantly without completely ceasing. The biggest disadvantage in their use is the addiction plus also a deshinbition badly mastered which may induce the passage to the suicidal act. However, the risk benefit remains favorable to the molecules because of their low toxicity. The French Agency for Drugs, following the representatives of the Special Interest Group on Psychopharmacology of the Royal College of Psychiatrists and the British Association for Psychopharmacology, examined these developments and recommended clinical practices. These different working groups re-examined the advantages and disadvantages of benzodiazepines in particular in the elderly and in bipolar patients. The British being much less severe than before. The synthesis of different opinions based on recent studies shows that benzodiazepines can be used in an intermittent way with minimal risks provided that patients are selected.
\end{abstract}

Keywords: Benzodiazepines; Anxiety; Sleep disorders; Dependence; Elderly patients

\section{Introduction}

Antidepressants are widely used in all forms of anxiety [1] but there is a therapeutic place for the use of benzodiazepines. Benzodiazepines (BZDs) are low-toxic drugs; their therapeutic index is very high. Doses that can cause fatal poisoning are much higher than therapeutic doses and never lead to death if other treatments are not combined. However, these drugs have adverse effects at therapeutic doses especially in the elderly population. Indeed, elderly patients have more side effects with BZDs than younger subjects due to pharmacokinetic and pharmacodynamics changes related to age. The main problem of prescribing BZDs is dependence [2]. The French Agency for Safety and Health Products (Afssaps) report published in 2012 has rekindled the debate over the use of benzodiazepines in the treatment of anxiety or sleep disorders. Indeed, 134 million boxes of drugs containing benzodiazepines or related drugs were sold in France in 2010 (50.2\% of anxiolytics and 37.6\% of hypnotics).

A. Each year, one in five French people consume at least one benzodiazepine or a related molecule;

B. $60 \%$ of benzodiazepine users are women;

C. Consumption increases with age;

D. Individual consumption of anxiolytic benzodiazepines has decreased in France by an average of $1.8 \%$ per year since 2002;
E. The consumption of hypnotic and related benzodiazepines is stable;

F. The consumption of benzodiazepines indicated either in the treatment of epilepsy or in the treatment of muscle contractures progresses. Given their indications, these drugs are not always perceived as benzodiazepines; Benzodiazepines are predominantly prescribed by general practitioners.

The consumption of benzodiazepines exposes them to a number of well-identified risks such as memory and behavioral problems. These risks are increased in the elderly. Regardless of age, the use of benzodiazepines also exposes them to a risk of abuse and psychological and physical dependence, with withdrawal syndrome when treatment is discontinued.

A. Different studies suggest a link between benzodiazepines and dementia. But the results of these studies are inconsistent. This issue is currently being evaluated by Afssaps.

B. There is a problematic use of benzodiazepines with diversion and misuse among addicts and a risk of criminal use for chemical submission.

C. Taking benzodiazepines like alcohol increases the risk of a road accident. 
On the other hand, comparisons of consumption between the countries of southern Europe (including France) are very unfavorable to the latter when they were established with AngloSaxon countries: $30 \%$ of the population over 65 In France, Spain in Portugal, $20 \%$ in Italy, against 5\% UK and USA. The aim of this article is to define the prescription conditions for the BZDs in order to optimize them, according to a British working group that has assessed the risk benefit of these molecules, which are largely attacked by Anglo-Saxons in the past but less nowadays [3].

\section{Use of BZDs in the Elderly}

Since BZDs are predominantly prescribed in the elderly, use in this population category will be considered in order to better understand the reasons $[4,5]$. The prevalence rates of BZD use differ widely among the populations studied and according to the definitions of the usual use in a given indication. In a cohort of 2792 subjects over 65 years old living in a retirement home in southwestern France, the prevalence rate of BZD use was 31.9\% [6]. The annual prevalence of BZDs prescriptions in Ontario for seniors decreased slightly from 1993 to 1998 (25.1\% versus $22.5 \%$ ). In elderly, the prevalence of BZDs increases with age (approximately $20 \%$ of those aged 65-69, approximately $30 \%$ of those over 85 years of age). There is a tendency for shortacting BZDs to be dispensed more than long-acting BZDs [7]. Despite the various clinical studies and observations, BZDs with long half-lives are still very used in the elderly. This leads to an increase in the risks in this population, particularly related to the accumulation of these drugs in the body.

Because of the heterogeneity of the definition of normal use and abuse, there are complicated problems concerning the interpretation of long-term risks and the benefits of using longterm risks and the benefits of using Of BZDs by the elderly. The categorization of the use of BZDs has been proposed as acute, intermittent, as well as short, long-term and continuous waiting to standardize the definitions of use [8]. Acute use lasts about 7 days or less. This use concerns treatments in emergency department before a psychotic agitation, during preoperative period (use where amnesia is desired), treatment of sleep disorders in hospital and treatment of alcohol withdrawal.

Intermittent use refers to the situation where BZDs are taken sporadically, usually two or three times a week, for periods not exceeding 60 to 90 days. It can also be referred to as a "long term" with intermittent use in cases where the treatment lasts 4 months or more [9]. Treatment of sleep and anxiety disorders by BZDs is usual in elderly subjects who often resort to intermittent use. When the use of BZDs is analyzed finely, it can be seen that the elderly patient often uses only low dosages in order to have a better morning activity. Thus, subjects taking doses of 0.5 to $1 \mathrm{mg}$ of lorazepam for supposedly facilitating sleep induction or decreasing their anxiety tell the doctor that the real benefit is to have a better morning comfort. Studies in healthy volunteers show that low doses of BZDs improve their psychometric performance $[10,11]$.

Continuous use means that the patient uses the medication every day. These subjects who take chronic anxiolytics suffer from anxiety disorders (generalized anxiety and/or insomnia). These elderly subjects and their prescribers continue to use these products in a chronic manner despite recommendations for short-term use. Among the usual users of BZDs, 21\% do so for their anxiolytic action, $17 \%$ for their hypnotic action. This corresponds to a prevalence rate of $3 \%$ of continuous use in the general population between 18 and 80 years [12]. Compared to subjects not using BZDs, continuous users are mostly older; it is often women taking these medicines since bereavement! Other indications are often indirect: follow-up of cardiovascular or rheumatologically disorders. $85 \%$ of continuous users have no support from health professionals [12].

Although no long-term efficacy studies of BZDs were conducted in elderly patients, tolerance was observed for diazepam and other BZDs after 22 weeks of treatment. Thus, after almost 6 months, most patients have a continuous treatment with stable dosage that can last for years. A single efficacy study was conducted in elderly subjects to assess the continued use of BZDs in the treatment of chronic insomnia [13]. This study shows that BZDs and behavioral treatments are very interesting for the treatment of insomnia in end-of-life subjects. After 24 months, the benefits for patients who were no longer taking the drug and no longer had behavioral therapy were completely lost, i.e. patients were at baseline. The long-term negative effects of the continued use of BZDs, apart from the risk of physical dependence, are unknown. Some studies have been carried out concerning secondary risks such as the risk of falls and fractures, or the reduction of cognitive impairments

\section{BZD Side Effects in the Elderly Population}

Elderly patients have more side effects with BZDs than younger subjects due to age-related pharmacokinetic and pharmacodynamics changes. Cognitive disorders appear to be the major side effects of BZDs [14]. Cognitive disturbances are characterized by anterograde amnesia, a decrease in the recall of short-term events and an increase in memory loss [15]. A followup study of 1389 people aged 60-70 showed that long-term use of BZDs was a risk factor for increased cognitive decline. Thus, authors [16] also showed that older women who used BZDs were at risk of declining physical performance. Subgroup analyzes indicated that the risk was greater when the dosages were higher than those recommended for long-term use. These results are confirmed by those of the Canadian Study of Health and Aging. In this work, the use of BZDs is associated with many symptoms that reflect altered cognitive functions [17]. This cognitive and physical decline suggests a potential relationship between the risk of falls and the use of BZDs.

The risk of hips' fracture has been shown to be increased in elderly BZDs patients. Patients appeared to be more vulnerable immediately after the onset of therapy than after several weeks 
of continuous use, probably that is linked to the tolerance phenomenon of sedation. BZDs with short half-lives do not seem safer than those with long half-lives. The latter showed that the presence of BZDs in plasma was not associated with an increased risk of fracture of the femoral neck except for lorazepam. In a recent review of the relationship between the use of BZDs and the risk of fracture of the femoral neck, the authors highlighted the differences in the various protocols that explained the contradictory results. Studies that do not show association were hospital studies, a type of study that lacks validity due to lack of adequate control group. However, Cumming et al conclude that there is epidemiological evidence strongly suggesting that the use of BZDs by the elderly increases their risk of cervical fractures by at least 50\% [18], other authors agree with over $40 \%$ of older adults presenting for emergency care after a fall had previously been prescribed BZD/Z drugs [19].

Another problem with the use of BZDs is the risk of dependence [20]. When BZDs are stopped abruptly, a withdrawal syndrome may occur [21]. The symptoms are mainly tremors, mental confusion, but as well anxiety and insomnia. Severe symptoms such as convulsions, psychotic manifestations may occur as well as a significant increase in blood pressure and myocardial ischemia in the event of abrupt arrest. Few weaning studies on elderly subjects have been performed; elderly patients compared with young adults have less severe withdrawal syndromes [22]. However, it has been observed that psychotic withdrawal reactions are more marked than in younger patients. Weaning is believed to be related to hyperactivity in neurotransmitting systems, like monoamines, that have been inhibited by the chronic administration of BZDs [23]. The fact that plasma concentrations of BZDs decreased more slowly may explain that withdrawal symptoms in the elderly are less severe. But, as well they are less related to BZDs by the patient, because they occur sometimes one week after the cessation of medication. It appears that the risks of withdrawal are increased particularly with the abrupt withdrawal of BZDs with a short half-life and rapid reduction of plasma concentrations, as well as high dosages, and long-term use. BZDs with a short or intermediate half-life may generate withdrawal symptoms at cessation, which occur between 24 and 36 hours after discontinuation, whereas BZDs with a long half-life may induce symptoms of weaning after practically a week and in this case the arrest of the BZDs is not always incriminated [24]. It appears that other factors may contribute to the severity of the withdrawal syndrome, such as a pre-morbid personality, and in particular passive-dependent personalities [25]. There are, of course, important physiological differences; thus prior alcohol consumption and low cultural level may increase withdrawal syndrome. All these results show that the benefit/risk of BZDs for the elderly is not clear. Given the high morbidity/mortality ratio associated with the use of BZDs in this population, Cumming et al conclude that elderly people should rarely be prescribed BZDs and that many of these patients taking these drugs can be weaned without problems in hospitals [18]. Several studies attempt to define withdrawal protocols in elderly patients. It was proposed a method based on a combination of cognitive-behavioral therapy and tapering prescribed BZDs [4]. Some researchers proposed replacement therapy with low doses of BZDs like lormetazepam which has a half-life of around 20 hours in the elderly [7]. This finding being made that proposing to elderly legitimate anxious people approaching the death of BZDs remains justified. Weaning from BZDs often requires hospitalization, which most of the time refuses: the patient, the family, and the hospital authorities given the daily prices. In fact, few drugs are studied with a suitable methodology (double-blind controlled) in the elderly (75 years and older). Drugs as serotonin reuptake inhibitors (SSRIs) are used in that population, but there are clinical trials showing their efficacy in patients more than 75 old.

\section{The Reasons for the Chronic Use of BZDs}

The reappearance of anxiety at the end of treatment, benzodiazepines hides anxiety, but do not make it disappear. Upon cessation of treatment, the patient feels disconcerted at the symptom that has disappeared and reappears. The difficulty for the prescriber is to make the difference between returning to initial anxiety or a rebound of anxiety. Thus the doctor and his patient are tempted to continue treatment without taking stock. The drug should be considered as a therapeutic aid and not as an element of "healing." In addition to unpleasant weaning, upon stopping the drug, the subject feels tired, excitable, has memory loss, obsessions, Aggressive behavior feels multiple pains (especially muscular) and can no longer sleep [26] On the other hand BZD use was associated with an increased risk of stroke among older individuals with Alzheimer 's disease [27].

The patient's first reflex is to take his benzodiazepine again. The doctor's question: whether these symptoms existed prior to treatment or not, to make the difference between the physical signs of anxiety and the signs of anxiety [28]. The fear of stopping treatment is also one of the reasons for chronic consumption, as well as the feeling of a certain inner peace, even a euphoria afforded by treatment [29]. Often the non-precision of the duration of the treatment by the doctor is the cause of overflows, as well as the almost automatic renewal of the prescription especially in the elderly patients. Theoretically, the prescriptions are limited but the prescriber is overwhelmed by the increase by the patient of his BZDs intake punctuated by the resurgence of anxiety linked to the phenomenon of quasi-daily weaning induced by the molecules with short half-life.

\section{Benzodiazepines and Memory}

It is essentially during a first intake of BZD that marked amnesia can appear. It is amnesia: especially anterograde, sometimes retrograde but reversible (semantic memory) compatible with the execution of complex activities of which the subject is not conscious and whose frequency is underestimated [30]. With the repetition of the doses a tolerance effect appears [31]: rarely complete and which does not prevent the occurrence 
of complications in particular cases (elderly subjects, association with other psychotropic agents, etc.).The tolerance with sedative effects occurs rapidly, and then amnesia tolerance appears [32].

There is a debate on whether long-term BZDs can promote cognitive decline and even increase the risk of Alzheimer's disease. A Swedish study [33] was showing that BZDs could have a protective effect against Alzheimer's disease by comparing chronic users of BZDs versus non-users over a 3-year period. This negative correlation persists after controlling for age, sex, education, use of non-steroidal anti inflammatory drugs and estrogens. In recent years a French research team has tended to prove the contrary [34]. These researchers have followed patients for 15 years and have seen an increased incidence of dementia cases using BZDs, but their results are highly controversial since it is difficult to say that in this cohort of elderly subjects [35]. Prescription of BZDs is nothing but a marker of entry into the disease.

\section{What Remains as the Scope of Use of the BZDs?}

Since the various side effects of BZDs, including dependence since the early 1980s, have been observed, research has been conducted to obtain rapidly acting anxiolytic agents. Selective serotonin reuptake inhibitors (SSRIs) display a delayed onset of action of several weeks, rendering their use in "reaction anxiety" obsolete. It is certainly the disinhibiting effect of the BZDs that is beneficial when the anxiety no longer allows the subject to act [36], but that can drive a person to commit suicide with impulsive subjects (alcoholic subjects, excited elderly people). The effect is paradoxical, which is not really the case, considering the known behavioral effects induced by benzodiazepines On the other hand, at high dosages, this effect disappears, replaced by sedative effect. The disinhibiting effect however may be the cause of attempted suicides sometimes successful if the subject then uses other drugs or other means of autolysis [37].

Apart from the elderly, a group of patients should hold our attention, bipolar patients. Indeed, BZDs can be considered an addictive substitute for alcohol, cannabis and other cocaine or opiates [38]. Bipolar patients may have to consume BZDs to avoid antidepressants for which the "honeymoon" is about two years. BZDs may be useful in sleep disorders induced by manic or hypo manic states. Benzodiazepines are generally not a "core" treatment for mania, but there use is done sometimes to no compliant bipolar patients. These drugs are able to rapidly help control certain manic symptoms such as restlessness, agitation, or insomnia, before anti manic drugs can take control. In all cases, BZDs can be prescribed safely in the short term (see below) and can be effective in many patients with "reaction" anxiety or sleep disorders. It should be noted that the BZDs lose some of their efficacy after one month of treatment insofar as a "down regulation" of the GABA A receptors is established [31], the patient must increase the dosage so that the action remains effective. Other psychotropic drugs have proved effective in the treatment of anxiety disorders including serotonin reuptake inhibitors (SSRIs). It is therefore necessary to weigh the benefit/ risk of the long-term treatment of an anxious pathology. There are also non-drug alternatives: cognitive-behavioral therapy and others alone or in combination with medications.

Dependence must be recognized as a major risk for treatment for more than one month, which is what should guide the prescription or not of a BZD [3]. If addiction is established for patients treated in the long term, it will be difficult to withdrawal these patients otherwise than by hospitalization. This is the reason why many elderly people continue to benefit from poorly adapted treatment by BZDs. Many patients are able to take BZDs for a short period of time provided the practitioner fully explains the issues. The corollary is therefore that they should not be prescribed if the minimum level of understanding is not reached.

\section{Referances}

1. Bourin M, Thibaut F (2013) A critical approach of the current treatment of anxiety disorders Current Psychopharmacology. Bentham Science Publishers 2(9): 104-112.

2. Park TW (2017) Debate: are benzodiazepines appropriate treatments for patients with substance use disorders? Yes. J Addict Med 11(2): 8789.

3. Baldwin DS, Aitchison K, Bateson A, Curran HV, Davies S, et al (2013) Benzodiazepines: risks and benefits. a reconsideration. J Psychopharmacol 27(11): 967-971.

4. Baillargeon L, Landreville P, Verreault R, Beauchemin JP, Grégoire JP, et al. (2003) Discontinuation of benzodiazepines among older insomniac adults treated with cognitive-behavioural therapy combined with gradual tapering: a randomized trial. CMAJ 169(10): 1015-1020.

5. Markota M, Rummans TA, Bostwick JM, Lapid M (2016) Benzodiazepine use in older adults: dangers, management, and alternative therapies. Mayo Clin Proc 91(11): 1632-1639.

6. Billioti de Gage S, Bégaud B, Bazin F, Verdoux H, Dartigues JF (2012) Benzodiazepine use and risk of dementia: prospective population based study. BMJ 345: e6231.

7. Petrovic M, Pevernagie D, Mariman A, Van Maele G, Afschrift M (2002) Fast withdrawal from benzodiazepines in geriatric inpatients: a randomised double-blind, placebo-controlled trial. Eur J Clin Pharmacol 57(11): 759-764.

8. Llorente MD, David D, Golden AG, Silverman MA (2000) Defining patterns of benzodiazepine use in older adults. J Geriatr Psychiatry Neurol 13(3): 150-160.

9. Kurko TA, Saastamoinen LK, Tähkäpää S, Tuulio-Henriksson A, Taiminen T, et al. (2015) Long-term use of benzodiazepines: Definitions, prevalence and usage patterns-a systematic review of register-based studies. Eur Psychiatry 30(8): 1037-1047.

10. Bourin M, Colombel MC, Malinge M (1995) Lorazepam 0.25mg twice a day improves aspects of psychometric performance in healthy volunteers. J Psychopharmacol 9(3): 251-257.

11. Bourin M, Colombel MC, Guitton B (1998) Alprazolam 0.125mg twice a day improves aspects of psychometric performance in healthy volunteers. J Clin Psychopharmacol 18(5): 364-372.

12. Dailly E, Bourin M (2008) The use of benzodiazepines in the aged patient: clinical and pharmacological considerations. Pak J Pharm Sci 21(2): 144-150.

13. Morin CM, Colecchi C, Stone J, Sood R, Brink D (1999) Behavioural and pharmacological therapies for late-life insomnia: a randomised controlled trial. JAMA 281(11): 991-999. 


\section{Open Access Journal of Neurology \& Neurosurgery}

14. Pomara N, Tun H, DaSilva D, Hernando R, Deptula D et al. (1998) The acute and chronic performance effects of alprazolam and lorazepam in the elderly: relationship to duration of treatment and self-rated sedation. Psychopharmacol Bull 34(2): 139-153.

15. Madhusoodanan S, Bogunovic OJ (2004) Safety of benzodiazepines in the geriatric population. Expert Opin Drug Saf 3(5): 485-493.

16. Fourrier A, Letenneur L, Dartigues JF, Moore N, Bégaud B (2001) Benzodiazepine use in an elderly community-dwelling population. Characteristics of users and factors associated with subsequent use. Eur J Clin Pharmacol 57(5): 419-425.

17. Murphy Y, Wilson E, Goldner EM, Fischer B (2016) Benzodiazepine use, misuse, and harm at the population level in canada: a comprehensive narrative review of data and developments since 1995. Clin Drug Investig 36(7): 519-530.

18. Cumming RG, Le Couteur DG (2003) Benzodiazepines and risk of hip fractures in older people: a review of the evidence. CNS drugs 17(11): 825-837.

19. Martinez-Cengotitabengoa M, Diaz-Gutierrez MJ, Besga A, BermúdezAmpudia C, López P (2017) Benzodiazepine prescriptions and falls in older men and women. Rev Psiquiatr Salud Ment. pii: S1888-9891(17): 30024-30031.

20. Tan KR, Rudolph U, Lüscher C (2011) Hooked on benzodiazepines: GABAA receptor subtypes and addiction. Trends Neurosci 34(4): 188197.

21. Bourin M, Thibaut F (2013) Benzodiazepines: tackling the symptoms of withdrawal. Neuropsychiatry 3: 263-265.

22. Soyka M (2017) Treatment of Benzodiazepine Dependence. N Engl Med. 376(12): 1147-1157.

23. Schweizer E, Case WG, Rickels K (1989) Benzodiazepine dependence and withdrawal in elderly patients. Am J Psychiatry 146(4): 529-531.

24. Griffin CE, Kaye AM, Bueno FR, Kaye AD (2013) Benzodiazepine pharmacology and central nervous system-mediated effects. Ochsner J 13(2): 214-223.

25. Lader M (1994) Anxiolytic drugs: dependence, addiction and abuse. Eur Neuropsychopharmacol 4(2): 85-91.

26. Bourin M, Vercelletto M (1999) Treatment of mood and behavioural disorders in Alzheimer's disease. Klinik Psikofarmakoloji Bülteni 9: 119-124.
27. Taipale $H$, Koponen $M$, Tanskanen A, Lavikainen $P$, Tolppanen AM et al. (2017) Use of benzodiazepines and related drugs is associated with a risk of stroke among persons with Alzheimer's disease. Int Clin Psychopharmacol 32(3): 135-141.

28. Shukla L, Bokka S, Shukla T, Kandasamy A, Chand P, et al. (2017) Benzodiazepine and "Z-Drug" Dependence: Data From a Tertiary Care Center. Prim Care Companion CNS Disord 19(1): 16 br02025.

29. Santos C, Olmedo RE (2017) Sedative-Hypnotic drug withdrawal syndrome: recognition and treatment. Emerg Med Pract 19(3): 1-20.

30. Beracochea D (2006) Anterograde and retrograde effects of benzodiazepines on memory. Scientific World Journal 16(6): 14601465.

31. Gravielle MC (2016) Activation-induced regulation of GABAA receptors: Is there a link with the molecular basis of benzodiazepine tolerance? Pharmacol Res 109: 92-100.

32. Bateson AN (2002) Basic pharmacologic mechanisms involved in benzodiazepine tolerance and withdrawal. Curr Pharm Des 8(1): 5-21.

33. Fastborm J, Forsell Y, Winblad B (1998) Benzodiazepines may have protective effects against Alzheimer disease. Alzheimer Dis and Assoc Disord 12(1): 14-17.

34. Lagnaoui R, Bégaud B, Moore N, Chaslerie A, Fourrier A, et al. (2002) Benzodiazepine use and risk of dementia: a nested case-control study. J Clin Epidemiol 55(3): 314-318.

35. Billioti de Gage S, Pariente A, Bégaud B (2015) Is there really a link between benzodiazepine use and the risk of dementia? Expert Opin Drug Saf 14(5): 733-747.

36. Bourin M (2003) Effets désinhibiteurs des benzodiazépines Encéphale, XXIX, S3-S7

37. Ginies G, Lamisse F, Gautier J, Choutet P, Breteau M, et al. (1978) Acute poisoning. A propos of 1200 cases. Sem Hop 54: 1130-1136.

38. Simon NM, Otto MW, Weiss RD, Bauer MS, Miyahara S, et al. (2004) Pharmacotherapy for bipolar disorder and comorbid conditions: baseline data from STEP-BD. J Clin Psychopharmacol 24(5): 512-520.

Your next submission with Juniper Publishers
will reach you the below assets
- Quality Editorial service
- Swift Peer Review
- Reprints availability
- E-prints Service
- Manuscript Podcast for convenient understanding
- Global attainment for your research
- Manuscript accessibility in different formats
( Pdf, E-pub, Full Text, Audio)
- Unceasing customer service
Track the below URL for one-step submission
https://juniperpublishers.com/online-submission.php

